\title{
The PANDEMYC Score. An Easily Applicable and Interpretable Model for Predicting Mortality Associated With COVID-19
}

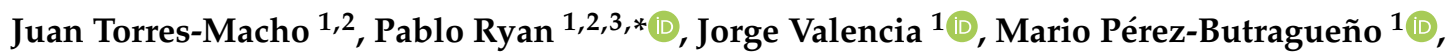

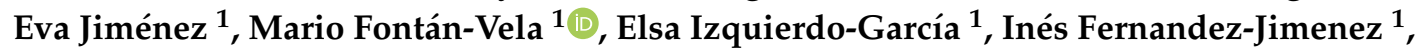 \\ Elena Álvaro-Alonso ${ }^{1}{ }^{1}$, Andrea Lazaro ${ }^{1}$, Marta Alvarado ${ }^{1}$, Helena Notario ${ }^{1}$, Salvador Resino ${ }^{4}$, \\ Daniel Velez-Serrano ${ }^{2,+}$ and Alejandro Meca ${ }^{5,+}$ \\ 1 University Hospital Infanta Leonor, 28031 Madrid, Spain; juan.torresm@salud.madrid.org (J.T.-M.); \\ jorge_vlr@yahoo.es (J.V.); marioperezb@hotmail.com (M.P.-B.); ejgonzalezbuitrago@salud.madrid.org (E.J.); \\ mario.fontan@salud.madrid.org (M.F.-V.); elsa.izquierdo@salud.madrid.org (E.I.-G.); \\ ifernandezj@salud.madrid.org (I.F.-J.); elenaalba.alvaro@salud.madrid.org (E.Á.-A.); \\ andrea.lazaro@salud.madrid.org (A.L.); marta.alvarado@salud.madrid.org (M.A.); \\ nelena.notario@gmail.com (H.N.) \\ 2 Department of Mathematics, Complutense de Madrid University (UCM), 28040 Madrid, Spain; \\ danielvelezserrano@mat.ucm.es \\ 3 Instituto de Investigación Sanitaria Gregorio Marañón (IiSGM), 28007 Madrid, Spain \\ 4 Unidad de Infección Viral e Inmunidad, Instituto de Salud Carlos III, 28007 Madrid, Spain; \\ Salvador.resino@gmail.com \\ 5 Department of Preventive Medicine \& Public Health, Rey Juan Carlos University, 28933 Madrid, Spain; \\ alalvaromeca@gmail.com \\ * Correspondence: pablo.ryan@salud.madrid.org \\ + Both authors contributed equally to this study.
}

Received: 18 August 2020; Accepted: 17 September 2020; Published: 23 September 2020

\begin{abstract}
This study aimed to build an easily applicable prognostic model based on routine clinical, radiological, and laboratory data available at admission, to predict mortality in coronavirus 19 disease (COVID-19) hospitalized patients. Methods: We retrospectively collected clinical information from 1968 patients admitted to a hospital. We built a predictive score based on a logistic regression model in which explicative variables were discretized using classification trees that facilitated the identification of the optimal sections in order to predict inpatient mortality in patients admitted with COVID-19. These sections were translated into a score indicating the probability of a patient's death, thus making the results easy to interpret. Results. Median age was 67 years, 1104 patients (56.4\%) were male, and $325(16.5 \%)$ died during hospitalization. Our final model identified nine key features: age, oxygen saturation, smoking, serum creatinine, lymphocytes, hemoglobin, platelets, C-reactive protein, and sodium at admission. The discrimination of the model was excellent in the training, validation, and test samples (AUC: $0.865,0.808$, and 0.883 , respectively). We constructed a prognostic scale to determine the probability of death associated with each score. Conclusions: We designed an easily applicable predictive model for early identification of patients at high risk of death due to COVID-19 during hospitalization.
\end{abstract}

Keywords: SARS-CoV-2; COVID-19; prediction score; mortality 


\section{Introduction}

Despite substantial efforts to prevent the spread of coronavirus 19 disease (COVID-19), at the end of June 2020 over 14 million people worldwide had tested positive for SARS-CoV-2, and more than 603,000 had died [1].

During March and April 2020, Spain had one of the highest rates of COVID-19 and had experienced one of the most severe outbreaks of the disease worldwide. The rate of infections in the Autonomous Region of Madrid has exceeded that of every other region in Spain, with more than $28 \%$ of all confirmed cases and a cumulative total of 42,747 hospitalized patients and 8441 deaths [2,3]. The excessive workload generated by the COVID-19 pandemic has led to drug shortages and an insufficient number of conventional and intensive care beds.

The wide variation in the symptoms of COVID-19 makes it difficult to predict the clinical course, thus complicating triage. Clinical experience has demonstrated significant heterogeneity in the course of severe acute respiratory syndrome coronavirus 2 (SARS-CoV-2) infection: while some patients are asymptomatic or progress with mild symptoms, others develop severe acute respiratory distress syndrome with multiorgan failure and death [4]. In addition, it is very difficult to accurately predict clinical outcomes in patients with such a myriad of clinical presentations.

Early detection of cases that are at high risk of progression to severe COVID-19 with an imminent risk of death and identification of contributing factors are now urgent and challenging. Accurate prediction of the mortality of COVID-19 would therefore enable targeted strategies that facilitate appropriate and early supportive care and enable patients to be categorized according to severity and prognosis. This is especially important in outbreaks, where prioritization of patients can reduce unnecessary or inappropriate use of health care resources [5].

We designed a reliable prognostic model using routine, widely available demographic, clinical, and laboratory data to predict mortality in patients hospitalized with COVID-19.

\section{Experimental Section}

\subsection{Study Design and Data Source}

We performed a retrospective observational study at Infanta Leonor University Hospital (ILUH), a secondary level hospital with 361 beds (including eight intensive care beds) in Vallecas, an area in the southeast of Madrid that is home to more than 305,000 inhabitants. Vallecas was one of the areas most affected by COVID-19 in the city of Madrid, with 4713 total confirmed cases as of July 7th, 2020 [3]. Therefore, the level of hospital saturation during the pandemic outbreak of COVID-19 was one of the highest in Spain. Consequently, in March, the hospital became a COVID-19 center, and all its healthcare professionals focused solely on infected patients.

The study population comprised all patients admitted to hospital with a confirmed diagnosis of COVID-19 based on a positive result in the SARS-CoV-2 reverse transcriptase-polymerase chain reaction assay between 2nd March and 31st May 2020. Samples were obtained via nasopharyngeal swabs.

In order to construct our predictive score, we collected demographic data, previous diseases, clinical presentation, and laboratory and radiological data at admission from electronic medical records and managed our findings using REDCap (Research Electronic Data Capture, Vanderbilt University, 2201 West End Ave, Nashville, TN 37235, USA) hosted at the Ideas for Health Association. REDCap is a secure, web-based software platform designed to support data capture for research studies [6].

\subsection{Ethical Aspects}

The study was approved by the Institutional Investigation and Ethics Review Board of Infanta Leonor University Hospital (CEI-ILUH) (Code ILUH R 027-20). Given the retrospective nature of the study, the need for informed consent from patients was waived. Data confidentiality was maintained at all times according to Spanish legislation. 


\subsection{COVID-19 Cohort Identification}

The primary cohort was divided into a training, a validation, and a test sample, with $40 \%, 30 \%$, and $30 \%$ of the observations reserved, respectively, for each.

\subsection{Model Development and Statistical Analysis}

In order to fit a logistic regression model, data were previously processed to avoid the effect of outliers, include missing values, and to consider possible non-linear associations between the target and the input variables.

During this preprocessing step we discretized the continuous variables into a specific number of categories and then generated a binary or dummy variable for each one. Discretization was performed using classification trees, one for each input. The aim was to identify cut-off points in the observed input values that discriminate as much as possible between the different target categories in order to enhance the predictive capacity of the transformed variables.

By definition, missing values and outliers are not present in binary variables. Moreover, the estimation of a value for each of these variables makes it possible to consider non-linear effects. The disadvantage of this approach is that a huge number of dummy variables can be generated. We solved this problem by applying a data transformation method that is widely used in the credit scoring field [7].

The method generates a new continuous variable (WoEx) for each input variable $X$. The values are obtained by averaging the target in the different categories obtained through the discretization process. This transformation procedure is usually known as the WoE (Weight of Evidence) transformation [8]. WoE measures the strength of an input variable for differentiating between the classes of the target variable. In this study, WoE measures the proportion of dead patients to live patients at each group level.

With $\mathrm{Y}$ as a binary dependent variable, $\mathrm{X}$ as an explanatory variable, and $\mathrm{X}_{\mathrm{c}}$ as the associated intermediate variable obtained after discretizing $X$ into the $X I$ attributes $x 1, x I$, the value of $W X$ at each category $x i$ is defined as:

$$
W_{X}\left(x_{i}\right)=\ln \left(\frac{P\left(Y=1 \mid X_{C}=x_{i}\right)}{P\left(Y=0 X_{C}=x_{i}\right)}\right)=\ln \left(O D D\left(x_{i}\right)\right)
$$

This value is derived by taking the logarithm transformation over the odds values associated with each of the categories $x_{i}$ derived from the original input variable.

WoE methodology overcomes previous selection problems and prevents the creation of an elevated number of dummy variables. Indeed, this approach generates only one transformed variable per input and, therefore, only one $p$-value when deciding whether or not to keep the variable in the regression model.

If a WoE variable, $W_{X}$, is included in the regression model, the odds ratios cannot be used to explain the effect of the original variable $X$ over the dependent variable, because $W_{X}$ is not measured in the same units as $X$. Therefore, a linear transformation of the value resulting from the multiplication of $W_{X}\left(x_{i}\right)$ and the regression coefficient estimated for $W_{X}$ is carried out. The aim of this transformation is to scale this value to a particular range of scores [9].

The score points are proportional to the logarithm of the predicted death/non-death odds of the patient. For each attribute $x_{i}$ of the variable, $\mathrm{X}$ is calculated as follows:

$$
\operatorname{SCORE}_{X}\left(x_{i}\right)=\left(-W_{X}\left(x_{i}\right) * \beta+\frac{\alpha}{n}\right) * \text { factor }+\frac{\text { offset }}{n}
$$

where

$W_{X}\left(x_{i}\right)$ is the weight of evidence of the ith attribute of the characteristic $\mathrm{X}$

$\beta$ is the regression coefficient associated with the variable $X$

$\alpha$ is the intercept of the logistic regression model 
$n$ is the number of variables in the logistic regression model

Factors and offset values are scaling parameters that enable the analyst to control the range of the scores and the rate of change in odds for a given increase in the score. The sum of the scores associated with each of the input variables provides a total score for the patient.

\subsection{Calibration}

For purposes of calibration, patients in the test database were split into deciles ordered by their probability of death. For each decile, the mean of the predicted probability of death was calculated and compared with the mean observed probability of death.

Moreover, to evaluate predictive capacity, the model was compared with other competitive machine learning methods. Based on the method described by DeLong et al. [10], these results were measured using the area under the curve (AUC) in the data test. The model that yielded the best results was the gradient boosting type. However, these are not significantly better in statistical terms than those obtained with the WoE methodology. In addition, those of the latter seem more reliable when generalizing to other samples, since the difference in the AUC obtained with the training and test tables is significantly lower than in the case of gradient boosting (Supplementary Table S1). Considering these observations and the difficulty in interpreting the results obtained with gradient boosting, we have chosen to consider the WoE methodology as the most appropriate to address a problem of this type.

The results are presented as the median (interquartile range) for continuous variables and as frequencies and percentages for categorical variables. Categorical data and proportions were analyzed using the chi-squared test or Fisher's exact test, as required. The Mann-Whitney test was used to compare continuous variables. In the case of multiple comparisons, a Bonferroni correction was applied to compare the results between the groups $(\alpha=0.05)$. The statistical analysis was performed using the statistical package R, foundation for Statistical Computing, Vienna, Austria. URL http://www.R-project.org/ version 3.1.1 (GNU General Public License) and SAS Enterprise Miner (SAS Institute, Cary NC, USA). All tests were two-tailed, and $p$-values $<0.05$ were considered significant.

\section{Results}

\subsection{Patient Cohorts}

The study population comprised 1968 patients. The baseline patient characteristics were as follows: median age was 67 (27) years, and 1104 patients (56.4\%) were male. A total of 325 patients died during hospitalization (17.7\%). Hypertension was the most frequent comorbidity (991 patients (51.8\%)), followed by dyslipidemia (36.8\%), smoking $(28 \%)$, and chronic heart disease $(21.7 \%)$. The most frequent symptoms at admission were fever $(75.1 \%)$, cough $(66.7 \%)$, and dyspnea $(56.8 \%)$.

Median oxygen saturation at admission was $94 \%$ (Interquartile Range, IQR=7) (Table 1).

Patients who died during hospitalization were older (median age, 82 vs. 63 years; $p<0.001$ ) and mainly male (68.7 vs. $54 \%$; $p<0.001$ ), with more comorbidities, such as hypertension, dyslipidemia, smoking/ex-smoking, and chronic heart disease. Dyspnea, cough, myalgia, headache, and low level of consciousness were more frequent in patients who died. These patients also had lower oxygen saturation (89 (11) vs. 95 (5); $p<0.001$ ).

When the cohort was divided into derivation and validation cohorts, we did not find significant differences in the distribution of the variables included in the model (Supplementary Table S2). 
Table 1. Demographics and comorbidity data of 1968 hospitalized patients with COVID-19 stratified by survival.

\begin{tabular}{|c|c|c|c|}
\hline Characteristics & $\begin{array}{c}\text { Alive } \\
n=1643\end{array}$ & $\begin{array}{c}\text { Dead } \\
n=325\end{array}$ & $p$-Value \\
\hline Male sex, No. (\%) & $882(54)$ & $222(68.7)$ & $<0.001$ \\
\hline Age, Median (IQR) & $63(26)$ & $82(16)$ & $<0.001$ \\
\hline Born in Spain, No. (\%) & $1132(72.8)$ & $281(89.2)$ & $<0.001$ \\
\hline Dead, No. (\%) & $0(0)$ & $325(100)$ & $<0.001$ \\
\hline \multicolumn{4}{|c|}{ Comorbid Conditions, No. (\%) } \\
\hline Chronic Heart Disease & $283(17.8)$ & $132(41.2)$ & $<0.001$ \\
\hline Hypertension & $765(48)$ & $226(70.6)$ & $<0.001$ \\
\hline Chronic Pulmonary Disease & $169(10.7)$ & $69(21.8)$ & $<0.001$ \\
\hline Asthma & $138(8.7)$ & $18(5.6)$ & 0.103 \\
\hline Stage 4 Chronic Kidney Disease & $68(4.3)$ & $42(13.2)$ & $<0.001$ \\
\hline Liver Cirrhosis & $22(1.4)$ & $9(2.8)$ & 0.099 \\
\hline Solid Neoplasm (Active) & $42(2.6)$ & $39(12.3)$ & $<0.001$ \\
\hline Hematologic Neoplasm (Active) & $21(1.3)$ & $13(4)$ & 0.001 \\
\hline HIV Infection & $11(0.7)$ & $0(0)$ & 0.284 \\
\hline Obesity & $239(17.8)$ & 45 (17.3) & 0.809 \\
\hline Diabetes & $337(21.2)$ & $99(31)$ & $<0.001$ \\
\hline Dyslipidemia & $347(33.8)$ & $97(53.6)$ & 0.001 \\
\hline Inflammatory Disease & $74(4.7)$ & $25(7.8)$ & 0.024 \\
\hline Dementia & $71(4.5)$ & $37(11.7)$ & $<0.001$ \\
\hline Malnutrition & $26(1.8)$ & $14(5.2)$ & 0.003 \\
\hline Smoker & $335(24.7)$ & $125(44.2)$ & $<0.001$ \\
\hline \multicolumn{4}{|c|}{ Current medications, No. (\%) } \\
\hline Non-Steroidal Anti-Inflammatory Drugs & $46(3.4)$ & $6(2.3)$ & 0.429 \\
\hline Angiotensin-Converting Enzyme Inhibitors & $339(22.1)$ & $93(29.8)$ & 0.002 \\
\hline Angiotensin II Receptor Blockers & $223(14.6)$ & $70(22.5)$ & $<0.001$ \\
\hline Inhaled Corticosteroids & $134(8.7)$ & 39 (12.6) & 0.033 \\
\hline Systemic Corticosteroids & $39(2.5)$ & $16(5.2)$ & 0.018 \\
\hline \multicolumn{4}{|c|}{ Vital signs at admission, median (IQR) } \\
\hline Temperature ${ }^{\circ} \mathrm{C}$ & $37(1.2)$ & $37.2(1.27)$ & 0.030 \\
\hline Heart Rate, Beats Per Minute & $89(21)$ & $89(24.2)$ & 0.849 \\
\hline Oxygen Saturation in Room Air, \% & $95(5)$ & $89(11)$ & $<0.001$ \\
\hline
\end{tabular}


Table 1. Cont.

\begin{tabular}{|c|c|c|c|}
\hline Characteristics & $\begin{array}{c}\text { Alive } \\
n=1643\end{array}$ & $\begin{array}{c}\text { Dead } \\
n=325\end{array}$ & $p$-Value \\
\hline \multicolumn{4}{|c|}{ Admission signs and symptoms, No. (\%) } \\
\hline Fever & $1197(75.7)$ & $225(71.9)$ & 0.206 \\
\hline Malaise & $652(41.7)$ & $140(45.6)$ & 0.281 \\
\hline Upper Respiratory Tract Symptoms & $353(22.5)$ & $68(22.1)$ & 0.879 \\
\hline Dyspnea & $850(54)$ & $221(70.4)$ & $<0.001$ \\
\hline Chest Pain & $163(10.4)$ & $20(6.4)$ & 0.042 \\
\hline Cough & $1070(68)$ & $188(60.1)$ & 0.015 \\
\hline Sputum Production & $187(11.9)$ & 45 (14.5) & 0.244 \\
\hline Hemoptysis & $29(1.8)$ & $4(1.3)$ & 0.653 \\
\hline Myalgia/Arthralgia & $349(22.3)$ & $25(8.1)$ & $<0.001$ \\
\hline Headache & $168(10.7)$ & $12(3.9)$ & $<0.001$ \\
\hline Altered Consciousness & $66(4.2)$ & $29(9.3)$ & $<0.001$ \\
\hline Seizures & $8(0.5)$ & $0(0)$ & 0.433 \\
\hline Abdominal Pain & $55(3.5)$ & $10(3.2)$ & 0.937 \\
\hline Vomiting/Nausea & $201(12.8)$ & $18(5.8)$ & 0.001 \\
\hline Diarrhea & $290(18.5)$ & $37(11.8)$ & 0.007 \\
\hline Skin Rash & $9(0.6)$ & $1(0.3)$ & 0.897 \\
\hline \multicolumn{4}{|c|}{ Laboratory findings, median (interquartile range) } \\
\hline Hemoglobin, G/L & $13.9(1.9)$ & $13(3.1)$ & $<0.001$ \\
\hline White Blood Cell Count, X10 $/ \mathrm{L}$ & $6480(3480)$ & $7760(5240)$ & $<0.001$ \\
\hline Lymphocyte Count - Cells/ $\mu \mathrm{L}$ & $1000(700)$ & $800(600)$ & $<0.001$ \\
\hline Neutrophil Count, Cells/ $\mu \mathrm{L}$ & $4800(3200)$ & $6200(4450)$ & $<0.001$ \\
\hline Hematocrit, \% & $41.5(6)$ & $39.2(8.8)$ & $<0.001$ \\
\hline Platelets, $\mathrm{X} 10^{9} / \mathrm{L}$ & $211(108)$ & $195(118)$ & $<0.001$ \\
\hline Activated Partial Thromboplastin Time & $25.9(3.8)$ & $27.2(5.1)$ & $<0.001$ \\
\hline International Normalized Ratio & $1.08(0.13)$ & $1.13(0.23)$ & $<0.001$ \\
\hline Aspartate Aminotransferase, U/L & $36(32)$ & $30(24.2)$ & $<0.001$ \\
\hline Alanine Aminotransferase, $\mathrm{U} / \mathrm{L}$ & $38(27)$ & $47(36.8)$ & $<0.001$ \\
\hline Glucose, mg/dL & $110(34)$ & $130(56)$ & $<0.001$ \\
\hline Creatinine, $\mathrm{mg} / \mathrm{dL}$ & $0.96(0.39)$ & $1.25(0.805)$ & $<0.001$ \\
\hline Sodium, mEq/L & $139(5)$ & $139(6)$ & 0.8761 \\
\hline Potassium, $\mathrm{mEq} / \mathrm{L}$ & $4.3(0.6)$ & $4.4(0.8)$ & 0.029 \\
\hline C-Reactive Protein, mg/L & $61.1(98.5)$ & $112(144)$ & $<0.001$ \\
\hline \multicolumn{4}{|c|}{ Radiology } \\
\hline Pathological chest $\mathrm{X}$-ray on admission, No. (\%) & $1424(91.2)$ & $287(91.4)$ & 0.478 \\
\hline
\end{tabular}

\subsection{Model Development}

The final model included the following variables: age; oxygen saturation at admission; smoking or ex-smoking; and serum creatinine, lymphocytes, hemoglobin, sodium, platelets, and C-reactive protein 
at admission. We used these variables to build the acronym PANDEMYC (Platelets, Age, Natremia, Kidney injury, Lymphopenia, Oxygen saturation, C-reactive protein) to name the score (Figure 1)

\begin{tabular}{|c|c|c|}
\hline \multicolumn{3}{|c|}{ PANDEMYC SCORE } \\
\hline 1. Age, y & Points & 5 Hemoglobin (g/dL) \\
\hline$<46$ & -46 & $<11.2$ \\
\hline $46-60$ & 4 & $11.2-12.2$ \\
\hline $61-78$ & 29 & $>12.2$ \\
\hline$>78$ & 50 & Missing \\
\hline Missing & 28 & 6. Smoking \\
\hline 2. $\mathrm{O}_{2}$ Saturation $(\%)$ & & No \\
\hline$<88^{2}$ & 59 & Yes \\
\hline $88-92$ & 36 & Missing \\
\hline $93-96$ & 21 & 7. C-reactive protein (mg/L) \\
\hline$\geq 97$ & -2 & $<9.7$ \\
\hline Missing & 30 & $9.7-65.9$ \\
\hline 3. Creatinine $(\mathrm{mg} / \mathrm{dL})$ & & $66-188.5$ \\
\hline$<0.77$ & 14 & $>188.5$ \\
\hline $0.77-1.08$ & 21 & Missing \\
\hline $1.08-1.71$ & 37 & 8. Platelets $\times 1000 / \mathrm{mm}^{3}$ \\
\hline$>1.71$ & 48 & $<124$ \\
\hline Missing & 29 & $>124$ \\
\hline 4. Lymphocytes $(\mu L)$ & & Missing \\
\hline$<500$ & 55 & 9. Sodium (mEq/L) \\
\hline $500-799$ & 37 & $<144$ \\
\hline $800-1000$ & 28 & $>144$ \\
\hline$>1000$ & 22 & Missing \\
\hline Missing & 19 & \\
\hline
\end{tabular}

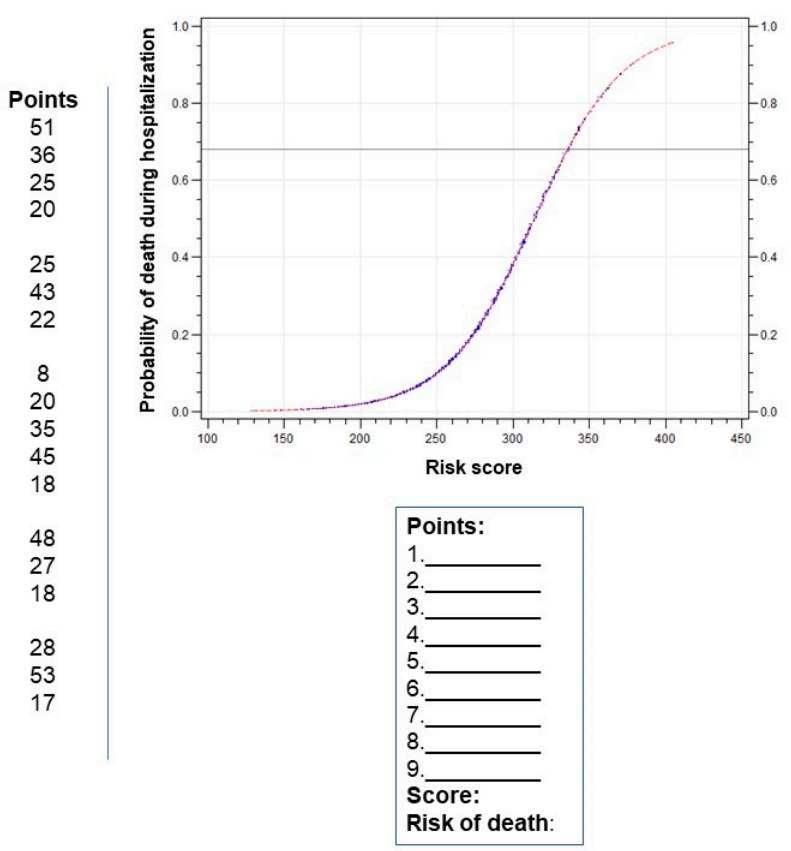

Figure 1. Resulting score for each variable during the discretization process. The mortality risk is calculated by summing the points for each variable.

\subsection{Validation}

To evaluate predictive capacity, the model was compared with other competitive machine learning methods (Table S1). The Transparent Reporting of a multivariable prediction model for Individual Prognosis or Diagnosis (TRIPOD) checklist was used to ensure the quality of our model (Supplementary Table S3).

Our model showed good predictive capacity in the training, validation, and test samples, with an AUC of $0.865,0.808$, and 0.883 , respectively. However, the presence of high AUC values did not mean that the individual probability of each class was well calibrated. Supplementary Figure S1 shows our probability calibration according to test data, which is good owing to the location of all points on the ideal line. When we compared our model with other models obtained using highly competitive machine learning methods such as gradient boosting, we did not find statistically significant differences (Supplementary Table S4).

\section{Discussion}

Several studies have reported risk factors associated with death in patients with COVID-19, although very few propose reliable prediction models, which should be constructed using an adequate sample size and a standardized methodology to avoid significant bias [5,11-14].

PANDEMYC showed high diagnostic accuracy (AUC $=0.88$ ), as reported elsewhere [15], revealed high-risk factors, and established a simple, intuitive, and quantitative method for accurately estimating the risk of death. The score is based on variables that are quickly and easily collected and reproducible in any hospital at admission (web page calculator www.pandemyc-score.com). This simple model enables us to prioritize patients, especially during a pandemic, when limited healthcare resources have to be allocated. It also makes it possible to select patients for early discharge or to establish more intensive monitoring and treatment [16]. For example, patients with a score $<200$ points and no significant respiratory insufficiency could be evaluated for early discharge. In our cohort, only two of 420 patients $(0.4 \%)$ with these characteristics died during hospitalization. 
All the factors included were clinically significant, with age, oxygen saturation, and serum creatinine at admission being the most relevant variables according to their weight and significance in the regression model. This observation is consistent with previous reports, thus showing the importance of aging, respiratory distress, and kidney injury as prognostic factors [12,14].

Analytical variables such as C-reactive protein, lymphopenia, and anemia indicate the association between the degree of inflammation and the risk for severe COVID-19 [15]. Thrombopenia is probably related to imbalance of the coagulation system and systemic thrombotic microangiopathy, which have been described in COVID-19 patients and are closely associated with mortality [17]. Finally, smoking is also a prognostic factor and may be associated with endothelial damage, as may other factors [18]. We did not detect significant values for variables that were frequently significant in previous models, such as gender and hypertension.

Despite the nature of the target classification variable, two main approaches are commonly distinguished for addressing classification problems, namely, interpretable methods and predictive methods [19]. The former typically place more emphasis on ensuring that the models obtained are easy to interpret, whereas the latter focus more on predictive accuracy. Consequently, the approaches are considered, at least to some extent, mutually exclusive, i.e., interpretability or predictive accuracy comes at the price of the other. We believe that interpretability is a key characteristic of any predictive model for a new disease, albeit with considerable caveats.

It is noteworthy that our model assigns a score to the missing values of each variable, thus creating a realistic model for real-life practice. Our model is not influenced by outliers, because they are not affected in the discretization process and therefore play no role in score assignment.

The main limitation of this study is that it was performed in a single center with no external validation. Nevertheless, the predictive capacity of the model was assured, because the AUC between the training and test samples, without the participation of the last in the adjustment process of the model, were very similar ( 0.865 and 0.883 respectively). Another limitation is that the level of saturation could have affected patient outcome. However, as saturation involves many components (available ICU beds, patient/physician ratio, new admissions, number of transfers to other hospital on a daily basis), we were unable to investigate it.

\section{Conclusions}

In conclusion, we designed a reliable, easily applicable prognostic score that can be applied in limited-resource settings to optimize management of patients hospitalize with COVID-19.

Supplementary Materials: The following are available online at http://www.mdpi.com/2077-0383/9/10/3066/s1, Table S1. Results obtained with different models in the training, validation, and test tables, ordered from the highest to lowest AUC in the test table; Table S2 Demographics and comorbidity data for 1968 patients hospitalized with COVID-19 stratified into 3 samples (training, validation, and test); Table S3. TRIPOD Checklist: Prediction Model Development and Validation; Table S4. Comparisons between the different models based on the AUC values. The DeLong method was used for the comparisons, and a Bonferroni correction was applied for the multiple comparisons; Figure S1. Probability calibration according to test data.

Author Contributions: Conceptualization: P.R., J.T.-M., A.M., D.V.-S.; Data curation: P.R., J.T.-M. Formal analysis: A.M., D.V.-S.; Investigation and methodology: P.R., J.T.-M., A.M., D.V.-S.; Project Administration: P.R., J.T.-M.; Supervision and visualization: P.R., J.T.-M.; Writing-original draft preparation: P.R., J.T.-M., A.M., S.R.; Writing-review and editing: P.R., J.T.-M., J.V., M.P.-B., E.J., M.F.-V., E.I.-G., I.F.-J., E.Á.-A., A.L., S.R., H.N., M.A., D.V.-S., A.M. All authors have read and agreed to the published version of the manuscript.

Funding: No funding was received for the present study. All authors declare that they have nothing to disclose.

Acknowledgments: We are extremely grateful to all the frontline ILUH staff, who worked with great humanity and dedication under enormous pressure. We also thank all the people who helped us to collect the data: Silvia Veleda Sánchez, Laura Zazo Morais, Raquel Ruiz Páez, Pernilla Seidi Tirado Zambrana, María Antonia Cabezas Quintario, María de los Ángeles Martínez Izquierdo, Fernando Manuel Sánchez Aranda, María Sonsoles Sánchez González, Iris Sánchez Egido, Carla Ferrero San Román, Virginia Del Rosario Rodríguez, Juan Gabriel Huertas Peña, Sonia Pérez González, Teresa Collazo Lorduy, Arantzazu Zurrido, Mario Velasco, Laura Serrano, Ester San Segundo, Carlos Domingo, Nuria del Val, Carlota Martín, Laura Salinas, Andrés Merino. We acknowledge the support of the following: Paz Arranz García, Rosalía De Dios Álvarez, Juan Rodríguez Moreno, Fernando Cava 
Valenciano, María Ángeles Rodríguez Martínez, Dulce Ramírez Puerta, Miguel Imaz Díaz, Julio Miguel Vila Blanco, María Carmen Pantoja Zarza.

Conflicts of Interest: The authors declare that they have no conflicts of interest.

\section{References}

1. World Health Organization (WHO). Coronavirus Disease (COVID-19) Situation Report-182. Available online: https://www.who.int/docs/default-source/coronaviruse/situation-reports/20200720-covid-19-sitrep182.pdf?sfvrsn=60aabc5c_2 (accessed on 20 July 2020).

2. Ministerio de Sanidad. Centro de Coordinación de Alertas y Emergencias Sanitarias. Actualización no 119. Enfermedad por el coronavirus (COVID-19). 28 May 2020. Available online: https:/www.mscbs.gob.es/en/profesionales/saludPublica/ccayes/alertasActual/nCovChina/documentos/Actualizacion_119_COVID-19.pdf (accessed on 6 June 2020).

3. Comunidad de Madrid. Transparencia. Covid-19-TIA por municipios y distritos de Madrid. Available online: https://datos.comunidad.madrid/catalogo/dataset/covid19_tia_muni_y_distritos (accessed on 1 June 2020).

4. Zhou, F.; Yu, T.; Du, R.; Fan, G.; Liu, Y.; Liu, Z.; Xiang, J.; Wang, Y.; Song, B.; Gu, X.; et al. Clinical course and risk factors for mortality of adult inpatients with COVID-19 in Wuhan, China: A retrospective cohort study. Lancet 2020, 395, 1054-1062. [CrossRef]

5. Wynants, L.; Van Calster, B.; Collins, G.S.; Riley, R.D.; Heinze, G.; Schuit, E.; Bonten, M.M.J.; Damen, J.A.A.; Debray, T.P.A.; De Vos, M.; et al. Prediction models for diagnosis and prognosis of covid-19 infection: Systematic review and critical appraisal. Br. Med. J. 2020, 369, 1328. [CrossRef]

6. Harris, P.A.; Taylor, R.; Thielke, R.; Payne, J.; Gonzalez, N.; Conde, J.G. Research electronic data capture (REDCap)-A metadata-driven methodology and workflow process for providing translational research informatics support. J. Biomed. Inform. 2009, 42, 377-381. [CrossRef] [PubMed]

7. Brown, L. Developing Credit Risk Models Using SAS Enterprise Miner. and SAS/STAT: Theory and Applications; SAS Institute Inc.: Cary, NC, USA, 2014; ISBN 978-1612906911.

8. Hand, D.; Henley, W. Statistical Classification Methods in Consumer Credit Scoring: A Review. J. R. Stat. Soc. 1997, 160, 523-541. [CrossRef]

9. Yap, B.; Ong, S.; Husain, N. Using data mining to improve assessment of credit worthiness via credit scoring models. Expert Syst. Appl. 2011, 38, 13274-13283. [CrossRef]

10. DeLong, E.; DeLong, D.; Clarke-Pearson, D. Comparing the Areas under Two or More Correlated Receiver Operating Characteristic Curves: A Nonparametric Approach. Biometrics 1988, 44, 837-845. [CrossRef] [PubMed]

11. Soto-Mota, A.; Garza, B.A.M.; Rodriguez, E.M.; Rodriguez, J.O.B.; Romo, A.E.L.; Minutti, P.A.; Loya, J.V.A.; Talavera, F.E.P.; Avila-Cervera, F.J.; Burciaga, A.N.V. The Low-Harm Score for Predicting Mortality in Patients Diagnosed with Covid-19: A Multicentric Validation Study. medRxiv 2020. [CrossRef]

12. Xie, J.; Hungerford, D.; Chen, H.; Abrams, S.T.; Li, S.; Wang, G.; Wang, Y.; Kang, H.; Bonnett, L.; Zheng, R.; et al. Development and External Validation of a Prognostic Multivariable Model on Admission for Hospitalized Patients with COVID-19. SSRN Electron. J. 2020. [CrossRef]

13. Yadaw, A.S.; Li, Y.-C.; Bose, S.; Iyengar, R.; Bunyavanich, S.; Pandey, G. Clinical predictors of COVID-19 mortality. medRxiv 2020. [CrossRef]

14. Parohan, M.; Yaghoubi, S.; Seraji, A.; Javanbakht, M.H.; Sarraf, P.; Djalali, M. Risk factors for mortality in patients with Coronavirus disease 2019 (COVID-19) infection: A systematic review and meta-analysis of observational studies. Aging Male 2020, 1-9. [CrossRef] [PubMed]

15. Gong, J.; Ou, J.; Qiu, X.; Jie, Y.; Chen, Y.; Yuan, L.; Cao, J.; Tan, M.; Xu, W.; Zheng, F.; et al. A Tool to Early Predict Severe 2019-Novel Coronavirus Pneumonia (COVID-19): A Multicenter Study using the Risk Nomogram in Wuhan and Guangdong, China. medRxiv 2020. [CrossRef]

16. Horby, P.; Lim, W.S.; Emberson, J.; Mafham, M.; Bell, J.; Linsell, L.; Staplin, N.; Brightling, C.; Ustianowski, A.; Elmahi, E.; et al. Recovery Collaborative Group. Effect of Dexamethasone in Hospitalized Patients with COVID-19: Preliminary Report. medRxiv 2020. [CrossRef]

17. Connors, J.M.; Levy, J.H. COVID-19 and its implications for thrombosis and anticoagulation. Blood 2020, 135, 2033-2040. [CrossRef] [PubMed] 
18. Zhao, Q.; Meng, M.; Kumar, R.; Wu, Y.; Huang, J.; Lian, N.; Deng, Y.; Lin, S. The impact of COPD and smoking history on the severity of COVID-19: A systemic review and meta-analysis. J. Med. Virol. 2020. [CrossRef] [PubMed]

19. Rudin, C. Stop explaining black box machine learning models for high stakes decisions and use interpretable models instead. Nat. Mach. Intell. 2019, 1, 206-215. [CrossRef] 